\title{
Internal transport barrier dynamics with plasma rotation in JET
}

\author{
P.C. de Vries 1), E. Joffrin 2,3), M. Brix 1), C.D. Challis 1), K. Crombé 4), B. Esposito 5), \\ N.C. Hawkes 1), C. Giroud 1), J. Hobirk 6), J. Lönnroth 7), P. Mantica 8), T. Tala 9) and \\ JET-EFDA Contributors to the Work Programme*
}

JET-EFDA Culham Science Centre, Abingdon, OX14 3DB, UK.

1) EURATOM/UKAEA Fusion Association, Culham Science Centre, Abingdon, OX14 3DB, UK.

2) JET-EFDA-CSU, Culham Science Centre, Abingdon, Oxfordshire, OX14 3DB, UK.

3) Association Euratom-CEA, Cadarache, F-13108, France.

4) Department of Applied Physics, Ghent University, Rozier 44, 9000 Gent Belgium

5) Associazione Euratom-ENEA sulla Fusione, Via E. Fermi 45, 00044 Frascati, Roma, Italy.

6) Max-Planck-Institut für Plasmaphysik, Euraton Association, 85748, Garching, Germany.

7) Association Euratom-Tekes, Helsinki University of Technology, PO Box 4100, Finland.

8) Istituto di Fisica del Plasma, EURATOM/ENEA-CNR Association, Milano, Italy.

9) Association Euratom-Tekes, VTT, PO Box 1000, 02044 VTT, Finland.

* Annex to F. Romanelli, Fusion Energy 2008 (Proc. 22st IAEA Conf., Geneve, 2008) IAEA Vienna.

\section{E-mail contact of main author: Peter.de.Vries@jet.uk}

\begin{abstract}
At JET the dynamics of Internal Transport Barriers (ITBs) has been explored by trying to decouple the effects of heating on one hand and torque on the other with the ultimate objective of identifying the minimum torque required for the formation of transport barriers. The experiments shed light on the physics behind the initial trigger for ITBs, which often shows to be linked to the shape of the q profile and magnetic shear, while the further development was influenced by the strength of the rotational shear. In discharges with a small amount of rotational shear ITBs were triggered, which suggest that the rotational shear is not the dominant factor in the triggering process. However, the subsequent growth of the barrier was limited if the rotational shear was too low at the time of triggering. This growth phase may be highly non-linear, with several possible positive feedback loops, such as the increases of the toroidal and poloidal component of the rotational shear caused by the ITB itself.
\end{abstract}

\section{Introduction}

Advanced Tokamak scenarios are proposed to provide the conditions for steady-state operations of ITER $[1,2,3,4]$. This scenario aims to operate at high pressure $\left(\beta_{\mathrm{N}} \sim 3\right)$ and modest plasma current ( $\left.\mathrm{I}_{\mathrm{p}} \sim 9 \mathrm{MA}, \mathrm{q}_{95} \sim 5\right)$ with a large bootstrap current providing a noninductive self-generated current. Internal transport barriers (ITBs) are considered as a candidate for enhancing the confinement and reaching these higher pressures.

Various physical mechanisms are thought to enable the formation of transport barriers in plasmas by causing a local suppression of turbulence. Because, these mechanisms may not necessarily act independently, the whole process may be an interplay of more than one mechanism, making it difficult to identify the physics roots behind ITB formation. Nevertheless, studies identified two factors that play a crucial role: magnetic and rotational shear $[5,6]$. It was found for example that ITBs form more easily in plasmas that have a particular current density profile and safety factor, q, profile, such that there exists a region of low or even negative magnetic shear, $r \cdot q / q$ [7], furthermore, the initial formation or triggering of ITBs, is often closely linked to detailed characteristics of the q profile [8]. The role of rotational shear, usually given as the shearing rate: $\omega_{\mathrm{ExB}}=\mathrm{RB}_{\theta} / \mathrm{B}_{\phi} \cdot \mathrm{d}\left(\mathrm{E}_{\mathrm{r}} / \mathrm{RB}_{\theta}\right) / \mathrm{dr}$, has been pointed out in experiments in JT-60U and TFTR which applied balance neutral beam injection (NBI) in order to create low torque plasmas with ITBs $[9,10]$. Here, $B_{\theta}$ and $B_{\phi}$ are the poloidal and toroidal magnetic field, respectively, and $E_{r}$ is the radial electric field. At JET internal transport barriers are usually formed in discharges with predominant NBI and 
this system provides a large toroidal torque and consequently a large rotational shear. Studies have shown the effect of rotational shear on ITBs in JET discharges with small but positive magnetic shear [11]. Experiments to produce ITBs with low torque have been performed previously by simply increasing the fraction of Ion Cyclotron Resonance Heating (ICRH). However, the variety of differences between the NBI and ICRH, such as the power deposition profiles, ion and electron heating fraction or current drive characteristics, adds to the difficulty in interpreting the experimental results [12].

In next step devices such as ITER the rotational shear induced by the NBI is not expected to be as high as in the present devices. In this context, the understanding of the ITB dynamics has been explored at JET by trying to decouple the effects of heating on one hand and torque on the other with the ultimate objective of identifying the minimum rotational shear required for the formation and development of broad ITB as required for the steady state scenario. Decoupling these two effects should also assist in separating the physics of the initial triggering of the barrier and its further development.

JET has developed several original experiments to study these aspects. First of all, JET has the unique ability to modify the amplitude of the toroidal magnetic field ripple. The toroidal field (TF) ripple has been shown to affect the toroidal rotation [13] and tuning the ripple may alter the shape of the rotation profile. In a second set of experiments, a specific ICRH heating scheme has been used, which couples to ${ }^{3} \mathrm{He}$ minority, providing ion heating close to the plasma centre with no excitation of $\mathrm{H}$ minority or $\mathrm{D}$ majority species. The toroidal momentum deposited in the plasma can be changed by varying the mixture of ICRH and NBI heating whilst keeping the total power to the ions and ion heat flux constant [14]. Both experiments enabled studies on the effect of rotational shear on transport barriers. The first part of this paper, section 2, will concentrate on the details of the TF ripple experiments with ITBs [15]. These observations will be compared with those obtained in the predominant ICRH heated plasmas in section 3. The focus is on ion barriers formed in plasmas with reversed central magnetic shear. Section 4 deals with the growth phase of the transport barriers in both experiments and the role played by toroidal and also poloidal rotational shear on this process, after which the conclusions will be summarized in the last section.

\section{Experiments using TF ripple}

Standard operations at JET are carried out with a set of 32 toroidal field coils, all carrying equal current. At JET it is possible to vary the toroidal field ripple amplitude by independently powering the 16 odd and 16 even-numbered coils [16]. The imbalance current between the two coil sets can be changed in a controlled way increasing the TF ripple amplitude, $\delta_{\mathrm{BT}}$, defined as the relative amplitude of the magnetic field variation: $\delta_{\mathrm{BT}}=\left(\mathrm{B}_{\max }{ }^{-}\right.$ $\left.\mathrm{B}_{\min }\right) /\left(\mathrm{B}_{\max }+\mathrm{B}_{\min }\right)$. The values quoted in the remainder of this paper, are the maximum ripple in the plasma which is usually found at the separatrix near the outboard mid-plane. For standard JET operations (with 32 coils) the TF ripple is $\delta_{\mathrm{BT}}=0.08 \%$.

The TF ripple breaks the axi-symmetry of the magnetic field and enhances particle losses, in particular energetic ions such as alpha particles created in fusion reactions and those injected by NB or accelerated by ICRH. These non-ambipolar ion losses will have a significant impact on the plasma rotation. The return current due to the ion losses, induces a $\mathrm{j} \times \mathrm{B}$ torque in counter-current direction that counteracts the NBI torque on the plasma, which is in co-current direction at JET. Increased levels of TF ripple have been found to reduce the plasma rotation in JET and even created regions where the plasma rotated in counter current direction [13]. Since the torque deposition profile of the NBI and that induced by the TF ripple are not the same, also rotation profile shape is modified. TF ripple has been used as a tool to tune the plasma rotation and rotational shear in typical scenarios which formed ITBs, utilizing optimised low and reversed central magnetic shear [15]. 
In figure 1, two discharges are shown with almost identical parameters, albeit different TF ripple amplitudes of $\delta_{\mathrm{BT}}=0.08 \%$ and $1.0 \%$, respectively. Since the TF ripple induces losses of NB injected particles, the applied auxiliary power for the discharge with the highest TF ripple (69668) is slightly higher, to correct for the ripple losses. The power loss fraction and the total absorbed power have been calculated using the JAEA Orbit Following Monte Carlo (OFMC) code [17]. For these scenarios it was found that typically $17 \%$ of the NBI power is lost with a TF ripple of $\delta_{\mathrm{BT}}=1.0 \%$. For standard JET operations with $\delta_{\mathrm{BT}}=0.08 \%$ these losses are negligible. Although the absorbed power is kept constant $\left(\mathrm{P}_{\mathrm{abs}}=14.5 \mathrm{MW}\right)$, the plasma rotation in both pulses was found to be significantly different. The main difference between the two examples is the much lower rotational shear in the case with the higher TF ripple (69668) as will be discussed in detail later.

The scenario shown in figure 1 applied a toroidal magnetic field, $\mathrm{B}=2.2 \mathrm{~T}$, and plasma current, $\mathrm{I}_{\mathrm{p}}=1.8 \mathrm{MA}\left(\mathrm{q}_{95}=4.2\right.$ ) and used a plasma configuration with an elongation of $\kappa=1.74$ and a low triangularity of $\delta=0.22$. Early heating is applied, during the current ramp-up phase, combined with a prelude of Lower Hybrid Current Drive (LHCD). This produces a q profile with reversed central magnetic shear as can be seen in the q profile contours in figure 1c. These measurements have been preformed by Motional Stark Effect (MSE) polarimetry. Within the uncertainties of the measurement one finds that the minimum $q$ value, $q_{\min }$, in the plasma is just below 2. So-called grand-cascades of Alfvén modes [18, 8] have been seen in discharge 69670 at $\mathrm{t}=3.8 \mathrm{~s}$ and another one at $\mathrm{t} \sim 5 \mathrm{~s}$, while for 69668 these modes are only seen at $\mathrm{t}=3.7 \mathrm{~s}$. The integer $\mathrm{q}$ values that appear in the plasma and trigger these modes may be identified by $\mathrm{q}=3$ and $\mathrm{q}=2$ for the two times in 69670 .

The presence of ITBs can be deduced from the local steepening of the temperature gradient. The temperature gradient can be expressed by a normalised value, $\rho^{*}{ }_{\mathrm{T}} \equiv \rho_{\mathrm{s}} / \mathrm{L}_{\mathrm{T}}$, i.e. the ratio of the ion Larmor radius at the sound speed and the temperature gradient length [19]. A transport barrier is said to be present if the $\rho^{*} \mathrm{~T}$ value exceeds the empirical threshold of the $\rho^{*}{ }_{T}>\rho^{*}{ }_{T}^{\text {crit }}=0.014$ [19]. In figure $1 \mathrm{c}$ the contours of the ion $\rho^{*} \mathrm{~T}$ are shown as a function of time and major radius. Soon after the switch on of NBI and ICRH, signs of a
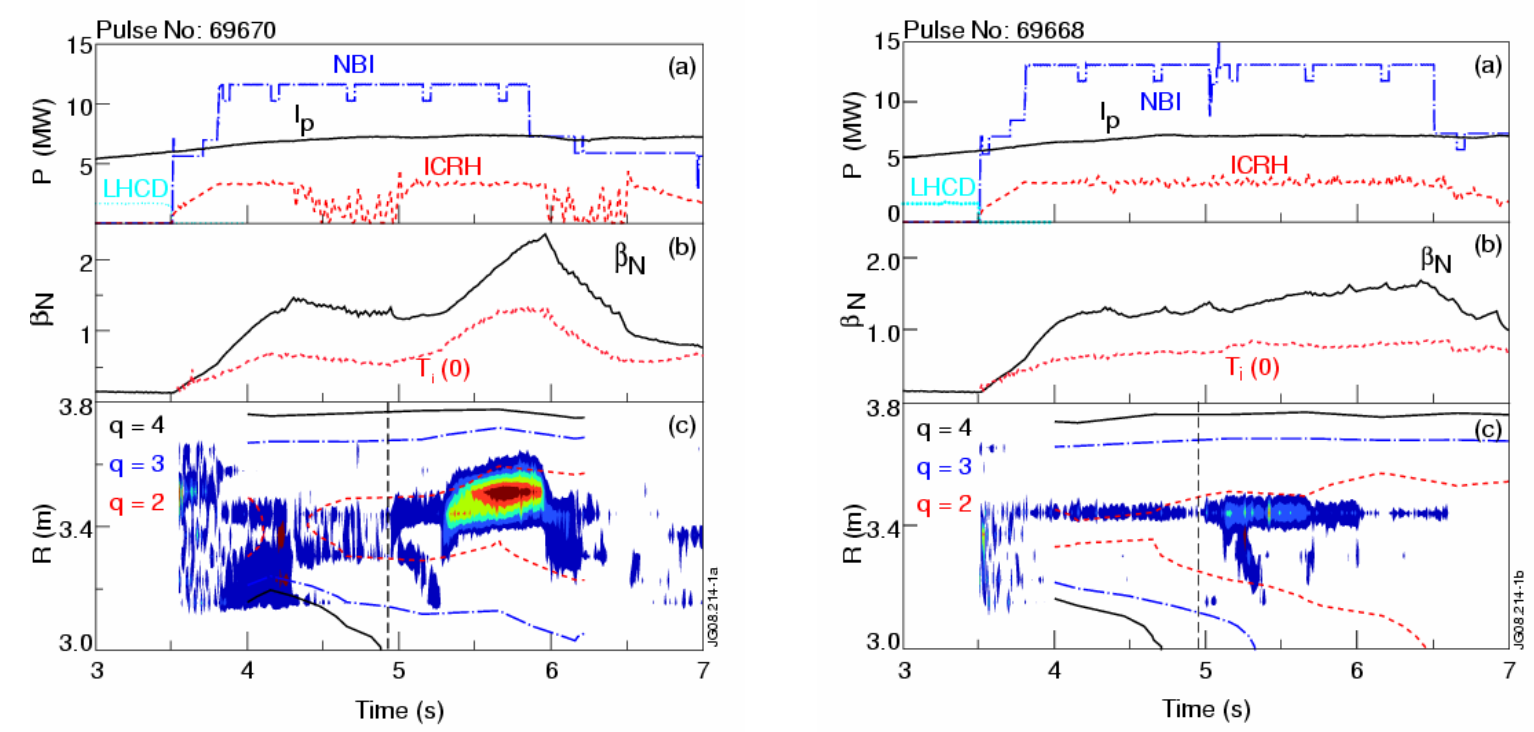

FIG. 1. a) NBI, ICRH and LHCD powers and the plasma current, $I_{p}$, in arbitrary units, for two discharges, 69670 (left) and 69668 (right), the first with a small, standard TF ripple of $\delta_{\mathrm{BT}}=0.08 \%$ and the second with a large ripple of $\delta_{\mathrm{BT}}=1.0 \%$, respectively. b) The central ion temperature and normalised $\beta$. c) The integer value contours of the q-profile, overlaid with the contours of the $\rho^{*}{ }_{T i}$, with in blue the minimum level $\rho^{*}{ }_{T}=\rho^{*}{ }_{T}^{\text {crit }}$ and in red the maximum level $\left(\rho^{*} T_{1}=0.050\right)$. The dashed lines give the time of the ITB trigger. 
temperature profile steepening are seen, especially in 69670. These are remnants of a barrier that formed during the LHCD prelude phase, located in the negative shear region, persisting briefly in the main experimental phase. In both discharges at approximately the same time ( $\mathrm{t}$ 5s), ITBs trigger (indicated by the dashed line in figure 1c). This coincides with an increase in the central ion temperature as shown in figure 1b. A weak outer and inner transport barrier can be seen, the latter moving radially inward. This signature, in the $\rho^{*} \mathrm{~T}$ contours, seems to be linked to the appearance of a rational q-surface in the plasma, and indeed a grand-cascade appears in 69670 quickly after the ITB trigger. This feature, identical to those observed in ref. [8, 22], cannot be predicted by transport models since usually no role is given to rational surfaces. On the other hand rarefaction of rational flux surfaces, i.e. the rarity of these surfaces, has been proposed to influence transport [20, 21]. It has been shown that zonal-flow structures can occur due to the gap in low order rational surfaces near integer q surfaces, causing modifications to the temperature profiles just after the minimum q drops below an integer value [23]. This would predict a steepening of the temperature profile to appear on both sides of the minimum q, after the $\mathrm{q}=2$ surface appears in the plasma, which is very similar to what is observed in these discharges.

Although ITBs are found to be triggered in both discharges, there is a striking difference in the subsequent development. In discharge 69668 the value of $\rho^{*}{ }_{\mathrm{T}}$ never exceeds 0.022 but in 69670 the outer barrier grows and reaches values of $\rho^{*}{ }_{\mathrm{T}}=0.065$ (see figure 1c). Because such strong barriers with large pressure gradients have been seen to trigger internal kink-modes and subsequent disruptions, the NBI power in this discharge is stepped-down prematurely in order to prevent this. The strength of the barrier is sufficient to significantly increase the central ion temperature and normalised $\beta, \beta_{N}$, as shown in figure $1 \mathrm{~b}$, while for the other discharge no such effect can be seen. As pointed out before, the difference between these two discharges is the TF ripple amplitude which has altered the rotational shear.

This has been explored further by carrying out a scan in TF ripple amplitude, keeping the scenario unchanged, applying the same level of absorbed power $\mathrm{P}_{\mathrm{abs}}=14.5 \mathrm{MW}$. ITB trigger events have been found in all four cases. However, as shown in figure 2a, the peak performance, expressed as $\beta_{\mathrm{N}}$, clearly decreases with TF ripple amplitude. At the highest TF ripple amplitude of $\delta_{\mathrm{BT}}=1 \%$ the performance improvement due to the barrier is only slightly better that that of a plasma without an ITB $\left(\beta_{\mathrm{N}} \approx 1.4\right)$.
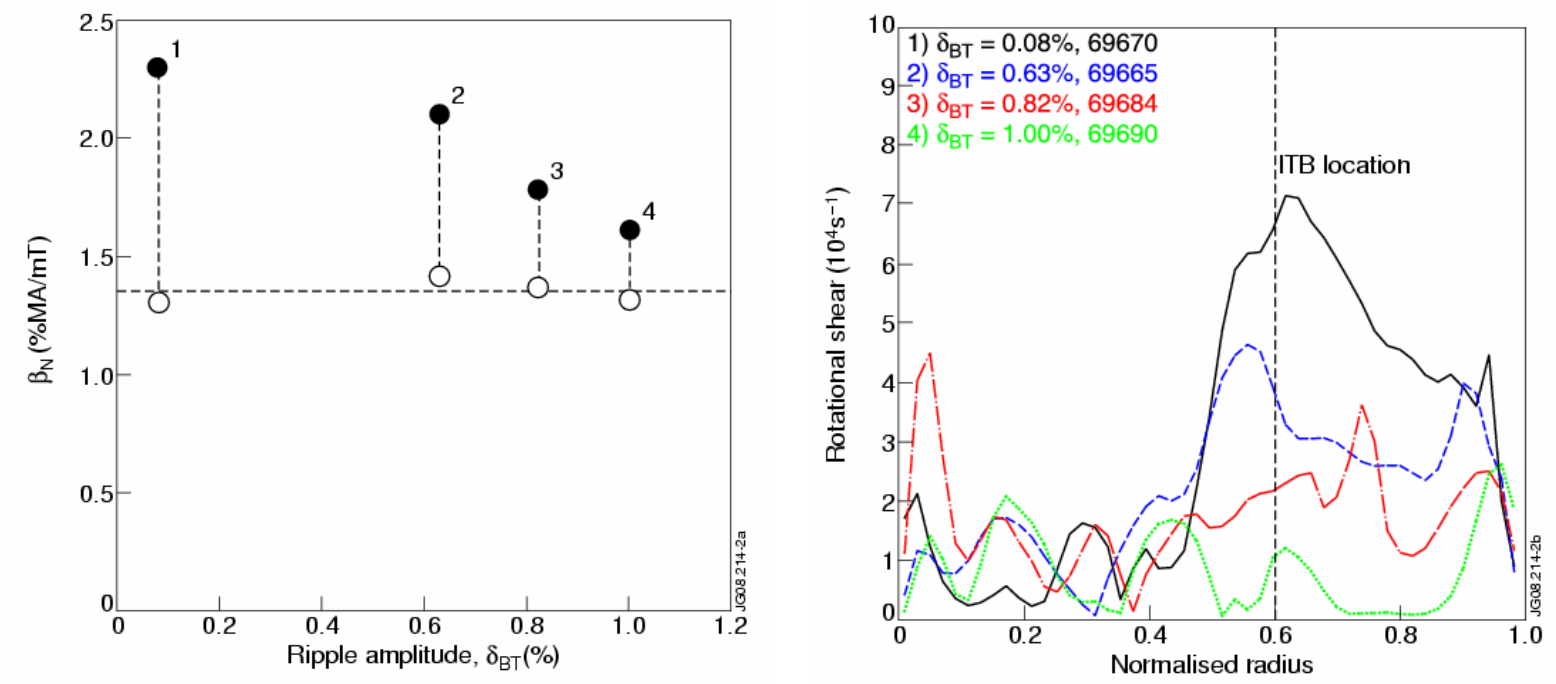

FIG. 2. a) The performance expressed as $\beta_{N}$, as a function of TF ripple amplitude, $\delta_{\mathrm{BT}}$, for a series of discharges with reversed central magnetic shear. The open circles and dashed line gives value before the ITB development. b) The rotational shearing rate at the time the ITBs are triggered, for the discharges shown in figure $2 b$. The dashed vertical line gives the approximate ITB position. 
Similar observations have been made for ITB scenarios with positive, zero or low magnetic shear [15]. Figure $2 \mathrm{~b}$ shows the respective rotational shear profiles, at the time the ITB is triggered, for the discharges from figure 2a. These profiles have been calculated under the assumption of neo-classical poloidal rotation as discussed in section 4. The TF ripple has clearly a strong effect on the rotational shear profiles. The strongest barriers are found to develop in discharges with a high shearing rate, such as 69670 which has $\omega_{\mathrm{ExB}}=7 \cdot 10^{4} \mathrm{~s}^{-1}$ near where the position of the ITB.

\section{Experiments with dominant ICRH}

Instead of using the TF ripple to alter the rotation properties of the plasma, low torque experiments with a dominant fraction of ICRH have been carried out. Two issues may complicate such experiments; Firstly, the amount of ICRH power at JET is limited and the coupling is often compromised in the presence of ELMs. Secondly, coupling of ICRH on D or minority $\mathrm{H}$ ions, mainly heats the electrons, while the aim of this experiment is to study ion ITBs which may be governed by different physics than those in the electron channel. At JET an ITB scenario has been developed that uses ICRH coupling to ${ }^{3} \mathrm{He}$ minority and predominantly heats the ions, as long as the ${ }^{3} \mathrm{He}$ minority concentration, which is controlled in real-time, is kept below 8\% [14]. At a magnetic field of $\mathrm{B}=3.45 \mathrm{~T}\left(\mathrm{I}_{\mathrm{p}}=2.5 \mathrm{MA}, \mathrm{q}_{95}=4.5\right)$, $37 \mathrm{MHz}$ ICRH provides central ion heating without coupling to other resonances. The high magnetic field also ensures a higher H-mode threshold and hence no or moderate ELMs, allowing good ICRH coupling up to 7MW. Similar to the scenario discussed in the previous section, a target q profile with reversed central magnetic shear is achieved by using a prelude of LHCD and early heating by NBI and ICRH, as shown in figure 3a. Figure 3c shows that ITBs are triggered, even with a large fraction of ICRH (69414) $\left(\mathrm{P}_{\mathrm{ICRH}}=6.1 \mathrm{MW}\right.$, $\mathrm{P}_{\mathrm{NBI}}=7.1 \mathrm{MW}$ ) and low toroidal torque of $7.4 \mathrm{Nm}$. The $\rho^{*}$ contours and detailed analysis of the temperature time traces show that barriers are triggered at two times, the first, probably when $\mathrm{q}_{\min }=3$ appears in the plasma and the second at $\mathrm{q}_{\min }=2$. No MSE $\mathrm{q}$ profile measurements were available, but those from a similar discharge suggest that $\mathrm{q}=2$ and 3 surfaces appear just before these barriers form. The first barrier triggered in 69414 at
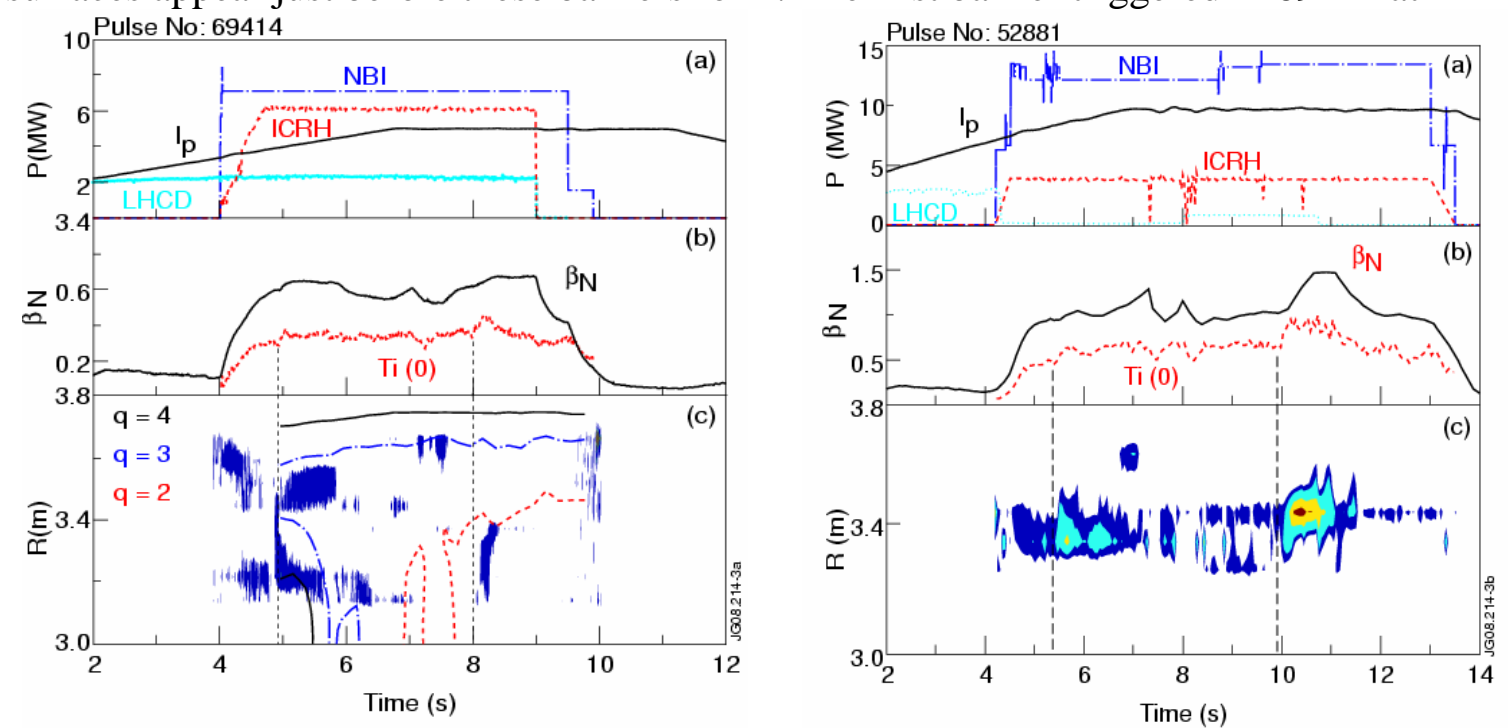

FIG. 3: a) NBI, ICRH and LHCD power and the plasma current, $I_{p}$, in arbitrary units, for two discharges, 69414 (left) and 52881 (right), the first with a dominant fraction of ICRH (He3 minority) and the second with predominant NBI heating. b) The central ion temperature, $T_{i}(0)$ and normalised $\beta$. c) The contours of the $\rho^{*} T_{T i}$, with in blue the minimum level $\rho^{*}{ }_{T}=\rho^{*}{ }_{T}^{\text {crit }}$ and in red the maximum level $\left(=\rho_{T}^{*}=0.050\right)$. In the left figure the q-profile contours for another, similar, discharge (69406) are shown for comparison. The dashed lines give the time of the ITB trigger. 
$t=5.95 \mathrm{~s}$, shows the signs of a similar double-barrier structure as discussed in the previous section. At $\mathrm{t}=8.0 \mathrm{~s}$ a grand-cascade, likely to be caused by $\mathrm{q}_{\min }=2$, is followed by the appearance of another small inner barrier. ITB trigger events have observed even in discharges with a toroidal torque as little as $3 \mathrm{Nm}$ and a rotational shear of $\omega_{\operatorname{ExB}} \sim 1 \cdot 10^{4} \mathrm{~s}^{-1}$ [14]. But both barriers in 69414 have little impact on the plasma performance, as shown in figure $3 \mathrm{~b}$ and the $\rho^{*}{ }_{\mathrm{T}}$ never reached above 0.021 .

If this is compared with a scenario of identical plasma parameters, but dominant NBI heating, strong ITBs are seen to develop. Such a case, discharge 52881, is shown in figure 3. The main plasma parameters are the similar as for 69414 except for the heating scheme. The input power is similar, although, slightly higher $\left(\mathrm{P}_{\mathrm{NBI}}=12-13 \mathrm{MW}\right.$ with $\mathrm{P}_{\mathrm{ICRH}}=3.7 \mathrm{MW} \mathrm{H}$ minority heating) but the main difference with 69414 is the torque of about $13 \mathrm{Nm}$. The barriers appear again at two times, presumably triggered again by the $q=3$ and $q=2$ surface appearing in the plasma, suggesting that the q profile characteristics are similar to those in 69414. The ITB reaches a peak strength of $\rho^{*} \mathrm{Ti}=0.045(\mathrm{t}=10.4 \mathrm{~s})$. This plasma exhibits a significantly higher central rotation frequency of $\sim 145 \mathrm{krad} / \mathrm{s}$ compared to $\sim 28 \mathrm{krad} / \mathrm{s}$ for 69414. The shearing rate at the time the ITB is triggered ( $t=9.93 \mathrm{~s})$ was found to be approximately $\omega_{\mathrm{ExB}} \sim 6 \cdot 10^{4} \mathrm{~s}^{-1}$.

\section{ITB growth and rotational shear}

Rotational shear is thought to suppress turbulence, by affecting the growth and radial extent of turbulent eddies. Since the radial force balance equation indicates a connection between the radial electric field and cross field heat transport, toroidal momentum transport and poloidal rotation, there are various positive feedback mechanism possible [5]. Hence, the strengthening of a transport barrier may yield further suppression of turbulence by for example enhancing the rotational shear suggesting a non-linear growth until neo-classical transport levels are reached.

Detailed studies have shown that in JET negative magnetic shear may result in electron barriers by providing stabilisation of Trapped Electron Modes (TEMs) [25]. Similarly, various models were used to calculate the linear growth rate, $\gamma$, of for example Ion Temperature Gradient (ITG) driven turbulence, which is thought to be responsible for anomalous ion heat transport. However, the triggering of ion ITBs in JET are usually not predicted from transport models or turbulence simulations [25, 26].

The values of rotational shear, as presented in figure $2 \mathrm{~b}$, have been calculated using the JETTO code and compared with the ITG growth rate, as discussed in ref. [11, 25], under the assumption of neo-classical poloidal rotation, determined by NCLASS [24]. The JET plasmas, discussed here, are in the low collisionality regime and it has been demonstrated that in this case the dominant contribution to the rotational shear is usually the gradient in the toroidal rotation profile $[11,13]$. These assumptions hold for plasmas without or with small barriers. The ratio of shearing rate to ITG growth rate, $\omega_{\mathrm{ExB}} / \gamma_{\mathrm{ITG}}$ was found to be always below unity. The ITB trigger events seen in these discharges seem to be independent of the level of $\omega_{\text {ExB }} / \gamma_{\text {ITG }}$. Nevertheless, in those discharges that form strong ITBs, such as those shown in figure 1 (69670) and 3 (52881), the initial values were high and close to unity ( $\left.\omega_{\mathrm{ExB}} / \gamma_{\text {ITG }} \sim 0.7-0.8\right)$. While for other discharges, shown in figure 1 (69668) and figure 3 (69414), the values are much lower, with values of $\omega_{\mathrm{ExB}} / \gamma_{\mathrm{ITG}}<0.1$ and $\omega_{\mathrm{ExB}} / \gamma_{\mathrm{ITG}} \sim 0.2$, respectively, at the time the ITB first appears.

After the ITB has been triggered, the barrier may enhance not only the temperature but also the toroidal rotation gradient, and is therefore able to further enhance the rotational shear. Furthermore, the assumption of neo-classical poloidal rotation may be invalid when 
the local temperature gradient length approaches the ion Larmor radius and this condition is reached in JET for strong ITBs. Poloidal rotation exceeding neo-classical values have been observed in JET plasmas with strong ITBs [27]. Figure 4 shows an example of rotation measurements with the JET Charge Exchange Spectroscopy (CXS) diagnostics. For the discharge 69670 (shown in figure 1), the poloidal and toroidal rotation profiles are shown, measured at the time the ITB triggers ( $\mathrm{t} \sim 4.75 \mathrm{~s})$ and when the barrier is strongest ( $\mathrm{t} \sim 5.75 \mathrm{~s})$. As expected the gradient in the toroidal rotation increases near the position of the barrier ( $\rho \sim 0.6)$ due to its effect on the momentum confinement further enhancing the toroidal component to the rotational shear. The poloidal rotation is, initially, close to the neoclassical values, however, when the ITB grows, it increases significantly. Poloidal rotation velocities 20 times the neo-classical value have been measured, peaking near the position of the barrier.

The enhanced poloidal rotation near the ITB region was found to have a large impact on the $\mathrm{E} \times \mathrm{B}$ shear and its component actually dominates over that of the toroidal rotation [28]. It could cause a 5 fold increase in shearing rate. As it seems that the amplitude of the poloidal rotation is linked to the strength of the barrier, its influence on the rotational shear is a non-linear component in the growth process. Hence, in plasmas with insufficient rotational shear to start with, a small 'seed-ITB', triggerd by for example the

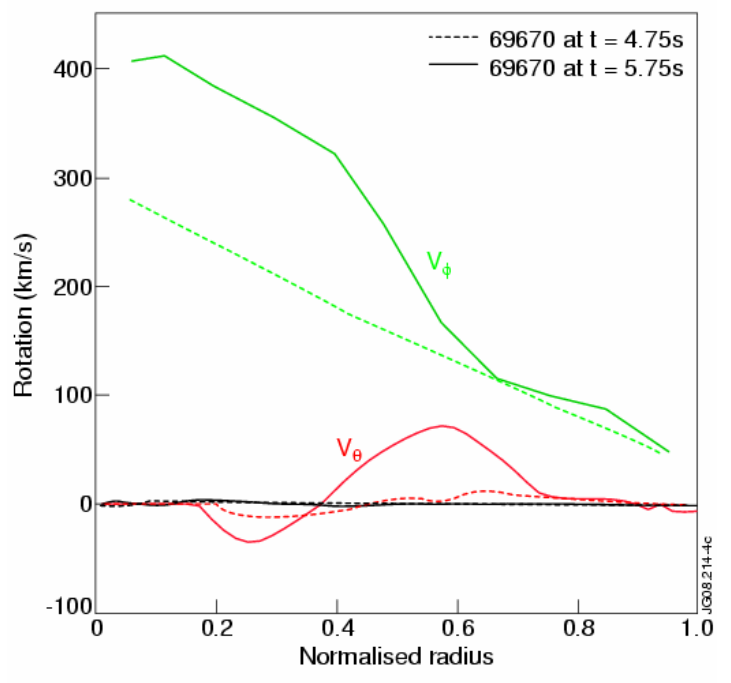

FIG. 4: The change in the toroidal (green), $V_{\phi}$, and poloidal (red), $V_{\theta}$, rotation profiles, as measured by the JET CXS diagnostics, from just prior to the ITB trigger until it is at its maximum strength. The poloidal rotation predicted by NCLASS is shown in black. specific characteristics of the q-profile, is not able to grow. In the other cases, however, the barrier may be able to push the sub-critical value $\omega_{\mathrm{ExB}} / \gamma_{\mathrm{ITG}}$ above unity, after which positive feedback is possible between the barrier strength, toroidal and poloidal rotational shear and stabilization of ITG driven turbulence, yielding a further growth of the barrier.

\section{Summary}

At JET the dynamics of internal transport barriers (ITBs) has been explored by trying to decouple the effects of heating on one hand and torque. This was achieved by tuning the plasma rotation and rotational shear in scenarios that form ion ITBs, by tuning either the TF ripple or the fraction of ICRH to NBI power.

The experiments shed light on the physics behind the initial trigger for ion ITBs, which at JET often show to be linked to the shape of the q profile and magnetic shear. In JET the rotational shear is usually found to be insufficient to trigger barriers, although it is well possible that this is the case in devices with a higher torque density. As described here, in plasmas with negative central shear and a low rotational shear, small, short-lived ion ITBs were triggered, which indicates that the overall rotational shear is not the dominant factor in this triggering mechanism. This does not mean that small scale changes in rotational shear or built-up of poloidal flow do not take part in this process. It shows that ITBs may be triggered in plasmas with a low torque and overall rotation such as in ITER. The subsequent growth of the internal transport barriers in JET, however, was affected by the rotational shear. A 'seedbarrier', triggered by the appearance of a rational q surface in the plasma, may be able to kick-start this process, as long as sufficient initial rotational shear and momentum flux is 
available. Only weak, transient ITBs were observed in discharges with a low torque and small rotational shear.

The improvement of transport in fusion plasmas is generally seen as a good thing, though the non-linear growth of the pressure gradient due to ITBs, may lead to unstable plasmas causing possibly a disruption of the discharge. Hence, control of the ITB growth should be understood. For the development of Advanced Tokamak scenarios the formation of wider, moderate but controllable barriers may be more favourable [29].

\section{Acknowledgements}

This research was funded partly by the United Kingdom Engineering and Physical Sciences Research Council and by the European Communities under the contract of Association between EURATOM and UKAEA. The views and opinions expressed herein do not necessarily reflect those of the European Commission. This work was carried out within the framework of the European Fusion Development Agreement.

\section{References}

[1] KIKUCHI, M, Nucl. Fusion 30 (1990) 265.

[2] LITAUDON, X., et al., Plasma Phys. Control. Fusion 44 (2002) 1057.

[3] LUCE, T.C., et al., Nucl. Fusion 43 (2003) 321.

[4] GREEN, B.J., et al., Plasma Phys. Control. Fusion 45 (2003) 687.

[5] BURRELL, K.H., Phys. Plasmas 4 (1997) 1499.

[6] CONNOR, J.W., et al., Nucl. Fusion 44 (2004) R1.

[7] CHALLIS, C.D., et al., Plasma Phys. Control. Fusion 43 (2001) 861.

[8] JOFFRIN, E., et al., Nucl. Fusion 43 (2003) 1167.

[9] SAKAMOTO, Y, et al., Nucl. Fusion 41 (2001) 865.

[10] SYNAKOWSKI, E.J, et al., Phys. Rev, Lett. 78 (1997) 2972.

[11] TALA, T., et al., Plasma Phys. Control. Fusion 43 (2001) 507.

[12] C. D. CHALLIS, et al., Plasma Phys. Control. Fusion 44 (2002) 1031.

[13] DE VRIES, P.C., et al., Nucl. Fusion 48 (2008) 035007.

[14] HAWKES, N.C., et al., "Ion Transport Barrier Formation with Low ExB Shear in JET", in Proc. of $34^{\text {th }}$ EPS Conf. on Plasma Phys. Control. Fusion (Warsaw, 2007).

[15] DE VRIES, P.C., et al., Plasma Phys. Control. Fusion 50 (2008) 065008.

[16] TUBBING, B.J.D and the JET team, 'Experiments with TF ripple in JET', in the Proc. of the $22^{\text {th }}$ EPS Conf. on Plasma Phys. Control. Fusion (Bournemouth, 1995) Vol. 19C, pIV001 (1995).

[17] SHINOHARA, K, et. al., Nucl. Fusion 43 (2003) 586.

[18] SHARAPOV, S.E., et al., Phys. Lett. A 289 (2001) 127.

[19] TRESSET, G., et al., Nucl. Fusion 42 (2002) 520.

[20] ROMANELLI, F, and ZONCA, F, Phys. Fluids B 5 (1993) 4081.

[21] GARBET, X, at al., Phys. Plasmas 8 (2001) 2793.

[22] AUSTIN, M.E., et al., Phys. Plasmas, 13 (2006) 082502.

[23] WALTZ, R.E., et al., Phys. Plasmas 13 (2006) 052301.

[24] HOULBERG, W., et al., Phys. Plasmas 4 (1997) 3231.

[25] BARANOV, Y.F., et al., Plasma Phys. Control. Fusion 46 (2004) 1181.

[26] TALA, T, et al., Nucl. Fusion 46 (2006) 548.

[27] CROMBÉ, K., et al., Phys. Rev. Letters 95 (2005) 155003.

[28] CROMBÉ, K., et al., 'Radial electric field during advanced tokamak scenarios on JET with toroidal field ripple', in the Proc. of $35^{\text {th }}$ EPS Conf. on Plasma Phys. Control. Fusion (Crete, 2008).

[29] RIMINI, F.G., et al., in the Proc. of this conference, EX/1-2. 